\title{
AVIFAUNA EN EL CAMPUS DE LA UNIVERSIDAD NACIONAL AGRARIA LA MOLINA (UNALM), LIMA - PERÚ
}

\section{AVIFAUNA OF UNIVERSIDAD NACIONAL AGRARIA LA MOLINA (UNALM) CAMPUS}

\author{
Fernando Takano Goshima ${ }^{1}$ y Nadia Castro Izaguirre ${ }^{2}$
}

\section{Resumen}

Se realizaron conteos mensuales de aves, entre Enero y Agosto de 2001, y avistamientos desde Setiembre de 2003 hasta Diciembre del mismo año, en las diferentes áreas del campus de la Universidad Nacional Agraria La Molina (UNALM, Lima). Como resultado se obtuvo una lista que incluye 46 especies vistas por lo menos en 3 ocasiones diferentes. La mayor parte de ellas pertenece al orden Passeriformes, mientras que dentro de las familias destacan Emberizidae, Columbidae y Psittacidae. Las especies más abundantes son Zenaida meloda, Columbina cruziana, Crotophaga sulcirostris y Dives warszewiczi. Las especies menos abundantes son Bubulcus ibis, Hirundo rustica, Myrtis fanny y Parabuteo unicinctus. Las especies de mayor distribución son Z. meloda, C. cruziana, D. warszewiczi, Troglodytes aedon y Pyrocephalus rubinus. Las especies de menor distribución son Bubulcus ibis, Icterus graceannae, Aratinga wagleri, Athene cunicularia y Glaucidium peruanum. Por otro lado, al incluir datos de avistamientos ocasionales fuera del período de estudio se observaron 13 especies raras o poco frecuentes en el campus. Dada esta gran diversidad de aves, el campus de la UNALM puede ser considerado como uno de los lugares urbanos más idóneos para observación de aves (birdwatching) dentro de la ciudad de Lima.

Palabras clave: conteos, avistamientos, lista, diversidad, UNALM

\begin{abstract}
Fieldwork consisting in monthly bird surveys - from January to August 2001- and observations of birds - from September to December 2003-, was conducted in all the area of the Universidad Nacional Agraria La Molina (UNALM) main campus. As a result, a list of 46 species, registered in at least 3 different occasions, was obtained. The majority of those birds belong to the order Passeriformes and among the families those with the greatest number of species are Emberizidae, Columbidae and Psittacidae. The most abundant species are Zenaida meloda, Columbina cruziana, Crotophaga sulcirostris and Dives warszewiczi, whereas the least abundant ones are Bubulcus ibis, Hirundo rustica, Myrtis fanny y Parabute unicinctus. The most widely distributed species are Z.meloda, C.cruziana, D. warszewiczi, Troglodytes aedon and Pyrocephalus rubinus. On the contrary, the least widely distributed species are Bubulcus ibis, Icterus graceannae, Aratinga wagleri, Athene cunicularia and Glaucidium peruanum. In addition, 13 rare or uncommon species were also registered, including data from occasional observations out of the study period. Due to its great diversity of birds UNALM main campus can be regarded as one of the best bird watching urban sites within Lima city.
\end{abstract}

Key words: surveys, observations of birds, list, diversity, UNALM

\section{Introducción}

Debido a la rápida expansión de las ciudades y a su efecto en la vida silvestre es necesario estudiar la biodiversidad urbana e incluir el conocimiento ecológico en el planeamiento urbano (Niemelä, 1999; Savard et al., 2000). En el caso de la ciudad de Lima, a pesar de la drástica expansión demográfica que ha experimentando en las últimas décadas (De Soto, 1986), los estudios publicados sobre aves urbanas son escasos (González, 2002; Ramírez \& González, 2001). En el campus de la Universidad Nacional Agraria La Molina (UNALM) una gran cantidad de alumnos ha llevado a cabo diversos estudios en las últimas décadas (Quinteros, 1992), pero la información no ha sido publicada formalmente y hasta el momento no se cuenta con una lista oficial de la avifauna que alberga dicho campus.

El presente trabajo constituye una recopilación de datos extraídos de 2 trabajos realizados de manera independiente, y tiene como objetivo principal presentar un listado de la avifauna del campus de la UNALM, además de describir brevemente algunos resultados sobre los patrones de abundancia y distribución de las especies. Asimismo, se espera que esta información pueda servir como referencia para futuros estudios sobre las aves urbanas. 


\section{Materiales y Métodos Área de estudio}

El lugar de estudio fue el campus de la Universidad Nacional Agraria La Molina (1206' S.; $76^{\circ} 57^{\prime}$ O.), localizado en la ciudad de Lima, a una altitud de 243.7 msnm y que corresponde, de acuerdo a Holdridge (1960), a la zona de vida llamada «desierto desecado subtropical» (dd-S). La temperatura anual promedio es de $20^{\circ} \mathrm{C}$, la humedad relativa promedio $84 \%$, y la precipitación anual 11.9 mm (según datos del Observatorio Meteorológico Alexander von Humboldt, UNALM).

El área aproximada del campus es de 220 ha y las instalaciones constan principalmente de aulas, edificios administrativos, laboratorios, zonas de esparcimiento, granjas de animales y zonas de cultivos. Además, destaca la presencia del jardín botánico Octavio Velarde Núñez, viveros de plantas ornamentales y un pequeño arboretum.

\section{Metodología}

Como se indicó anteriormente, esta nota reúne información obtenida, a partir de 2 trabajos diferentes realizados por los autores dentro del campus de la UNALM. En el primero se estimó la abundancia relativa de las aves mediante conteos mensuales entre los meses de Enero y Agosto de 2001, utilizando el método de transecto en línea. Los 55 transectos recorridos se encontraban distribuidos en todo el campus de la UNALM y sumaban una longitud total de $13 \mathrm{~km}$. La velocidad de recorrido fue de 1.5 $\mathrm{km} /$ hora y se registraron todos los individuos que estuvieran a una distancia de hasta $30 \mathrm{~m}$ a cada lado de la línea de observación. El horario para los conteos fue entre las 7:00 y 9:30 a.m. de los días sábados (día laboral con el menor número de alumnos en el campus) y estuvieron a cargo de 4 observadores y sus respectivos anotadores. Sólo se realizaron registros visuales (no se incluyeron registros por cantos) y se tomó nota tanto de las especies como del número de individuos.

El segundo trabajo de campo se llevó a cabo entre Setiembre y Diciembre de 2003. Para este caso, el mapa del campus fue dividido en 45 zonas (ver anexo), en base a algunos límites físicos como avenidas, jardines, caminos entre campos de cultivo, granjas, etc. En cada una de las zonas se realizaron avistamientos semanales, en las mañanas (entre 7:00 y 8:00 a.m.) y en las tardes (entre 4:00 y 6:00 p.m.). Se registraron las especies presentes en cada zona, identificadas ya sea por observación directa o por canto (sin tomar en cuenta la abundancia).

En ambos casos se utilizaron binoculares 8 x 40 ó 10 x 50 y para la determinación de especies se utilizaron las guías de aves de Koepcke (1964) y Clements \& Shany (2001).

Además se realizaron observaciones ocasionales fuera del período de estudio, entre los años 2004 y 2005.

\section{Análisis de datos}

La lista de especies fue elaborada en base a los registros obtenidos en ambos trabajos de campo, principalmente (2001 y 2003). Únicamente se incluyeron las especies que fueron registradas por 3 observadores diferentes como mínimo, en distintas ocasiones. Se elaboró además una lista de especies raras que incluye datos de ambos trabajos y datos de observaciones ocasionales fuera del período de estudio, donde figuran las especies que fueron observadas en menos de 3 ocasiones. Ambas listas se encuentran clasificadas según los criterios taxonómicos de la guía de Clements \& Shany (2001).

La abundancia de las especies fue estimada a partir de los datos de los conteos (primer trabajo de campo). Las especies más abundantes fueron definidas como las que presentaron el mayor número de individuos registrados en los 8 conteos mensuales. La distribución de las especies fue estimada a partir de su presencia o ausencia en las diferentes zonas de avistamiento (segundo trabajo de campo). Las especies de mayor distribución fueron aquéllas cuya presencia fue registrada en la mayor cantidad de zonas.

Se incluyeron además algunos datos de las observaciones ocasionales fuera del período de estudio (entre los años 2004 y 2005), pero no se contó con estos datos para elaborar la lista principal de especies (Tabla 1).

\section{Resultados}

Luego de integrar la información de los conteos y avistamientos se obtuvo una lista de 46 especies, dentro de 21 familias y 9 órdenes (Tabla 1). El orden con mayor número de especies es Passeriformes con 22 especies, seguido por Psittaciformes y Columbiformes (Figura 1). Por su parte, las familias más diversas son Emberizidae, Columbidae y Psittacidae (Figura 2).

Entre las especies más abundantes destacan Zenaida meloda, Columbina cruziana, Crotophaga sulcirostris y Dives warszewiczi. En contraste, las de menor abundancia fueron Bubulcus ibis, Hirundo rustica, Myrtys fanny y Parabuteo unicinctus (Tabla 2).

Por otro lado, Z. meloda, C. cruziana, D. warszewiczi, Troglodytes aedon y Pyrocephalus rubinus presentaron una mayor distribución, en tanto que Bubulcus ibis, Icterus graceannae, Aratinga wagleri, Athene cunicularia y Glaucidium peruanum se encuentran entre las de menor distribución. En la Tabla 3 se muestra tanto el número de zonas en los que se registraron las especies, como la frecuencia de los registros de cada una según el total de zonas del mapa en el anexo.

Asimismo, al incluir información de otros avistamientos hasta el año 2005 se observaron 13 especies raras o poco frecuentes en el campus tales 
como Egretta caerulea, que fue vista una sola vez. Otras especies raras son Pandion haliaetus, Falco peregrinus y Poospiza hispaniolensis (Tabla 4).

Tabla 1. Lista de aves del campus de la UNALM basada en registros hechos durante el período 2001-2003.

\begin{tabular}{|c|c|c|c|c|}
\hline & Nombre científico & Nombre común & Familia & Orden \\
\hline 1 & Bubulcus ibis & Garza bueyera & Ardeidae & Ciconiiformes \\
\hline 2 & Coragyps atratus & Gallinazo cabeza negra & Cathartidae & \multirow{4}{*}{ Falconiformes } \\
\hline 3 & Circus cinereus & Gavilán de campo & \multirow{2}{*}{ Accipitridae } & \\
\hline 4 & Parabuteo unicinctus & Gavilán acanelado & & \\
\hline 5 & Falco sparverius & Cernícalo & Falconidae & \\
\hline 6 & Burhinus superciliaris & Huerequeque & Burhinidae & \multirow{2}{*}{ Charadriiformes } \\
\hline 7 & Charadrius vociferus & Chorlo doble collar & Charadriidae & \\
\hline 8 & Columba livia & Paloma doméstica & \multirow{5}{*}{ Columbidae } & \multirow{5}{*}{ Columbiformes } \\
\hline 9 & Zenaida auriculata & Rabiblanca & & \\
\hline 10 & Zenaida meloda & Cuculí & & \\
\hline 11 & Columbina cruziana & Tortolita peruana & & \\
\hline 12 & Metriopelia ceciliae & Cascabelita & & \\
\hline 13 & Aratinga wagleri & Loro frente roja & \multirow{5}{*}{ Psittacidae } & \multirow{5}{*}{ Psittaciformes } \\
\hline 14 & Aratinga erythrogenys & Loro máscara roja & & \\
\hline 15 & Psilopsiagon aurifrons & Periquito cordillerano & & \\
\hline 16 & Forpus coelestis & Periquito esmeralda & & \\
\hline 17 & Brotogeris versicolurus & Pihuicho & & \\
\hline 18 & Crotophaga sulcirostris & Guardacaballo & Cuculidae & Cuculiformes \\
\hline 19 & Glaucidium peruanum & Paca-paca & \multirow{2}{*}{ Strigidae } & \multirow{2}{*}{ Strigiformes } \\
\hline 20 & Athene cunicularia & Lechuza de los arenales & & \\
\hline 21 & Amazilia amazilia & Amazilia costeña & \multirow{4}{*}{ Trochillidae } & \multirow{4}{*}{ Apodiformes } \\
\hline 22 & Rhodopis vesper & Picaflor cola orquillada & & \\
\hline 23 & Thaumastura cora & Picaflor de Cora & & \\
\hline 24 & Myrtis fanny & Picaflor de Fanny & & \\
\hline 25 & $\begin{array}{l}\text { Camptostoma } \\
\text { obsoletum }\end{array}$ & Mosqueta silbadora & \multirow{3}{*}{ Tyrannidae } & \multirow{22}{*}{ Passeriformes } \\
\hline 26 & Pyrocephalus rubinus & Turtupilín & & \\
\hline 27 & Tyrannus melancholicus & Pepite & & \\
\hline 28 & $\begin{array}{l}\text { Pygochelidon } \\
\text { cyanoleuca }\end{array}$ & Santa Rosita & \multirow[t]{2}{*}{ Hirundinidae } & \\
\hline 29 & Hirundo rustica & Golondrina migratoria & & \\
\hline 30 & Troglodytes aedon & Cucarachero & Troglodytidae & \\
\hline 31 & Mimus longicaudatus & Chisco & Mimidae & \\
\hline 32 & Passer domesticus & Gorrión europeo & Passeridae & \\
\hline 33 & Carduelis magellanica & Jilguero cabecinegro & Fringillidae & \\
\hline 34 & Coereba flaveola & Pielerito & Coerebidae & \\
\hline 35 & Conirostrum cinereum & Mielerito gris & \multirow{2}{*}{ Thraupidae } & \\
\hline 36 & Thraupis episcopus & Violinista & & \\
\hline 37 & Volatinia jacarina & Saltapalito & \multirow{6}{*}{ Emberizidae } & \\
\hline 38 & Sporophila simplex & Espiguero simple & & \\
\hline 39 & Sporophila telasco & Espiguero corbatón & & \\
\hline 40 & Catamenia analis & Corbatita pico de oro & & \\
\hline 41 & Sicalis flaveola & Botón de oro & & \\
\hline 42 & Zonotrichia capensis & Gorrión americano & & \\
\hline 43 & Sturnella bellicosa & Huanchaco & \multirow{4}{*}{ Icteridae } & \\
\hline 44 & Dives warszewiczi & Tordo grande & & \\
\hline 45 & Molothrus bonariensis & Tordo parásito & & \\
\hline 46 & Icterus graceannae & Chiroca & & \\
\hline
\end{tabular}

Asimismo, en el parque «El Olivar» (distrito de San Isidro), uno de los lugares más visitados por los observadores de aves en la ciudad, se han registrado sólo 29 especies (Anónimo, 2005). Por lo tanto, dada la gran diversidad de aves del campus de la UNALM, éste puede ser considerado como un refugio de vida silvestre y, por ende, uno de los lugares más apropiados para la observación de aves (birdwatching) dentro de la ciudad de Lima.

Las especies más abundantes del campus - Zenaida meloda, Columbina cruziana, Dives warszewiczi y Crotophaga sulcirostris - son también muy comunes en otras áreas de la ciudad de Lima (González et al., 1998). En años anteriores, Z. meloda y C. cruziana han sido consideradas especies plagas en el campus, debido a su dominancia en los zonas de cultivo y al daño que causan a los cultivos de maíz (Quinteros, 1992). Asimismo, D. warszewiczi es también una especie asociada a los agroecosistemas del campus y destaca por su comportamiento granívoro (Quinteros, 1992).

Con respecto a algunas de las especies con menor abundancia, Rhodopis vesper $\mathrm{y}$ Thaumastura cora tampoco son comunes en otras áreas de la ciudad de Lima y dentro del campus fueron observadas principalmente cerca de los viveros de plantas ornamentales. Otra especie dentro de esta categoría es Passer domesticus, cuya distribución se limita a las zonas cercanas a las granjas, caballerizas y a la facultad de Ciencias Forestales. En las zonas mencionadas existen construcciones con tejados o techos de plancha corrugada, que aparentemente son ideales para proteger sus nidos. En el caso de Psilopsiagon aurifrons, su baja abundancia podría ser un reflejo de

\section{Discusión}

El campus de la UNALM presenta una alta riqueza de especies en comparación a otras áreas de la ciudad de Lima. Por ejemplo, en un estudio de un año de duración se registraron únicamente 34 especies en el «Lima Golf Club» (distrito de San Isidro; $12^{\circ}$ 4' S. y $77^{\circ} 2$ ' O.), considerada el área verde más grande de Lima (48 ha), que cuenta con alrededor de 2000 árboles y 3000 arbustos (Ramírez \& González, 2001). su comportamiento migratorio, pues sólo se observa en el campus durante algunos meses del año. Lo mismo ocurre con Hirundo rustica, que solamente fue observada en Enero de 2001, y es considerada como visitante de verano en la costa de Lima (Koepcke, 1964).

Z. meloda fue la especie de mayor distribución en el campus. Esta especie no es sólo común en los campos de cultivo, sino que también está muy 
habituada a la presencia humana y está presente en las zonas próximas a las aulas, edificios administrativos, etc. A pesar de que C. cruziana está ampliamente distribuida en el campus, cabe destacar que sólo se concentra en grandes grupos en lugares específicos, ya sea cerca de las granjas de vacunos y porcinos, o en lugares descampados con mucha tierra seca, donde suele forrajear, descansar o tomar baños de tierra. D. warszewiczi es también una especie muy común y conspicua. Es muy fácil verla y oírla en casi cualquier zona del campus, a diferencia de Troglodytes aedon que por su tamaño y comportamiento escurridizo no siempre es fácil de observar — prefiere zonas arbustivas, en las que sea fácil esconderse-, a pesar de ser muy ubicua. Otra especie muy común y conspicua es Pyrocephalus rubinus, que puede ser apreciada tanto en su fase melánica como en su fase normal.

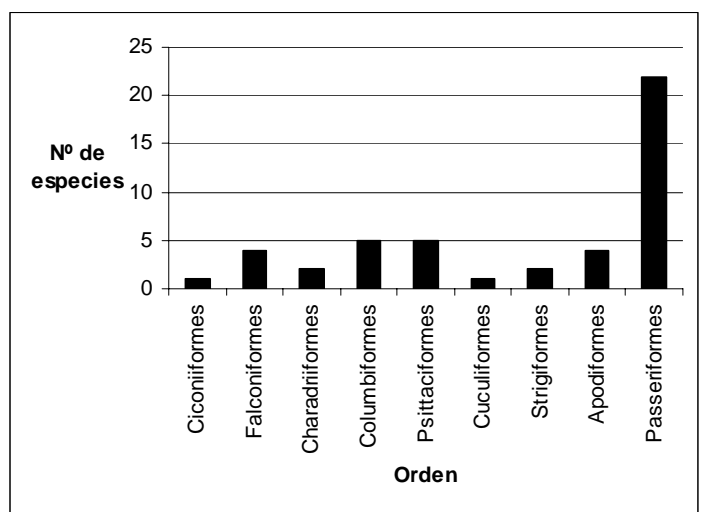

Figura 1. Número de especies por orden
Otras especies son especialistas con respecto al tipo de hábitat aunque su área de distribución pueda ser amplia, por ejemplo Sturnella bellicosa y Zonotrichia capensis pueden ser observados solamente en las chacras o cerca de ellas, Charadrius vociferus se encuentra en zonas de barbecho y Burhinus superciliaris prefiere áreas de poca vegetación o barbechos. En el caso de $Z$. capensis sucede algo curioso, pues aunque es un ave muy común en casi todas las áreas edificadas de Lima, ésta se ausenta en las áreas más edificadas de la UNALM y prefiere los campos de cultivo o las zonas más alejadas de la gente.

También existen especies de distribución amplia, pero que pueden ser encontradas en grupos densos sólo en lugares específicos, por ejemplo Molothrus bonariensis y Coragyps atratus suelen agruparse cerca de las granjas de vacunos y porcinos.

Es interesante señalar además que especies otrora introducidas como Coereba flaveola (González, 2002), Forpus coelestis, Aratinga erythrogenys o Thraupis episcopus (Koepcke, 1964) son ahora bastante comunes dentro del campus y se han adaptado aparentemente bien a los recursos del mismo.

En cuanto a las especies raras, todas las que aparecen listadas han sido reportadas previamente en el departamento de Lima, pero son poco frecuentes en la ciudad. Egretta caerulea, por ejemplo, es propia de lagunas y zonas pantanosas, y se considera una especie rara en nuestra región costera (Koepcke, 1964). Sporophila obscura es un ave solitaria y rara en el departamento de Lima (Koepcke, 1964) y su presencia, así como la de las otras especies del género Sporophila, se limitó a los campos de cultivo del campus.

Los individuos de la especie Circus cinereus han sido observados en varias oportunidades sobrevolando el campus, pero no se sabe si utilizan los recursos del área o si se reproducen en la misma. Es necesario,

Figura 2. Número de especies por familia

Por otro lado, la presencia de Bubulcus ibis se limitó a la zona de granjas, pues esta especie busca estar cerca del ganado donde abunden insectos (Koepcke, 1964). Aratinga wagleri y Athene cunicularia también mostraron una distribución restringida, pero estuvieron próximas a edificios administrativos y aulas. Glaucidium peruanum prefiere lugares arbolados (Koepcke, 1964) y en este caso fue observado en el arboretum $y$ en zonas próximas a éste. entonces, realizar estudios más detallados sobre estas especies.

\section{Conclusiones}

- En el período comprendido entre los años 2001 y 2003 se tiene una lista de 46 especies -registradas al menos en 3 ocasiones por observadores diferentes-, que es un número relativamente alto en comparación con otras zonas urbanos con grandes extensiones de áreas verdes como «Lima Golf Club» y el parque «El Olivar». Sin embargo, el número de especies es mayor si se incluyen 
aquellas consideradas como raras y las que se registraron en los años 2004 y 2005 (Tabla 4). De estas especies la mayoría pertenece a las familias Emberizidae, Columbidae y Psittacidae.

- Se pueden encontrar tanto especies nativas como introducidas, de las cuales algunas son raras en otros ambientes de Lima metropolitana (e.g. Psilopsiagon aurifrons o Charadrius vociferus).

- De las 46 especies listadas, las de mayor abundancia son Zenaida meloda, Columbina cruziana, Crotophaga sulcirostris y Dives warszewiczi, y las menos abundantes son Bubulcus ibis, Hirundo rustica, Myrtis fanny y Parabuteo unicinctus. Por otro lado, las especies de mayor distribución son Z. meloda, C. cruziana, D. warszewiczi, Troglodytes aedon y Pyrocephalus rubinus, y las de menor distribución son Bubulcus ibis, Icterus graceannae, Aratinga wagleri, Athene cunicularia y Glaucidium peruanum.

\section{Recomendaciones}

Debido a que la riqueza, abundancia y distribución de la avifauna en cualquier ecosistema varía con el tiempo, se recomienda continuar con los censos y avistamientos, pues permitirían detectar los cambios en la comunidad de aves de la UNALM. De esta manera, los datos registrados sobre la avifauna de la UNALM podrán ser útiles como información básica en el estudio de las aves urbanas.

\section{Agradecimientos}

Agradecemos especialmente a la Bióloga Martha Williams de Castro, a Luis Rico Llaque, y a todos los estudiantes y bachilleres que participaron tanto en los censos como en los avistamientos.

\section{Literatura citada}

Anónimo. 2005. (en línea). Lista de aves del Olivar de San Isidro. $<$ http:// www.avesdelima.com/lista_parques.htm>. Acceso 15 de diciembre de 2005.

Clements J. \& Shany N. 2001. A Field Guide to the Birds of Peru. Ibis Publishing Company, California, EEUU.

De Soto H. 1986. El Otro Sendero. Editorial El Barranco, Lima, Perú.

González O. 2002. Distribución y Dispersión del Mielerito (Coereba flaveola, Aves: Coerebidae) en la Ciudad de Lima, Perú. Ecología Aplicada. 1(1): 115-116.

González O., Pautrat L. \& Gonzalez J. 1998. Las Aves más Comunes de Lima y Alrededores. Editorial Santillana, Lima, Perú.

Holdridge L. 1960. Zonas de Vida Natural en el Perú. Memoria explicativa sobre el Mapa Ecológico del Perú. Instituto Interamericano de Ciencias Agrícolas de la OEA. Zona Andina.

Koepcke M. 1964. Las Aves del Departamento de Lima. Koepcke, Lima.

Niemelä J. 1999. Ecology and Urban Planning. Biodiversity and Conservation. 8: 119-131.
Quinteros Z. 1992. Determinación de los Patrones de Uso Temporal y Espacial de los Cultivos de Maíz por las Aves Granívoras en el Campo de la UNALM. Tesis para optar el título de Bióloga. Universidad Nacional Agraria La Molina, Lima.

Ramírez D. \& González O. 2001. Análisis de dos Métodos de Muestreo para el Cálculo de la Diversidad de Especies de Aves Terrestres en la Ciudad de Lima, Perú. Biota. 100: 114-123.

Savard J., Clergeau P. \& Mennechez G. 2000. Biodiversity Concepts and Urban Ecosystems. Landscape and Urban Planning. 659: 1-12.

Tabla 2. Abundancia de especies entre Enero y Agosto de 2001.

\begin{tabular}{|c|c|c|}
\hline Especie & $\begin{array}{c}\mathrm{N}^{\mathrm{o}} \\
\text { individuos }\end{array}$ & $\begin{array}{c}\text { Promedio } \\
\text { mensual }\end{array}$ \\
\hline Zenaida meloda & 255 & 36.43 \\
\hline Columbina cruziana & 183 & 26.14 \\
\hline Crotophaga sulcirostris & 126 & 18.00 \\
\hline Dives warszewiczi & 122 & 17.43 \\
\hline Volatinia jacarina & 97 & 13.86 \\
\hline Zenaida auriculata & 97 & 13.86 \\
\hline Pygochelidon cyanoleuca & 95 & 13.57 \\
\hline Troglodytes aedon & 92 & 13.14 \\
\hline Pyrocephalus rubinus & 89 & 12.71 \\
\hline Sturnella bellicosa & 75 & 10.71 \\
\hline Coereba flaveola & 55 & 7.86 \\
\hline Sporophila simplex & 48 & 6.86 \\
\hline Falco sparverius & 47 & 6.71 \\
\hline Sporophila sp. & 46 & 6.57 \\
\hline Mimus longicaudatus & 38 & 5.43 \\
\hline Coragyps atratus & 35 & 5.00 \\
\hline Sporophila telasco & 34 & 4.86 \\
\hline Zonotrichia capensis & 30 & 4.29 \\
\hline Camptostoma obsoletum & 28 & 4.00 \\
\hline Molothrus bonariensis & 28 & 4.00 \\
\hline Thraupis episcopus & 24 & 3.43 \\
\hline Carduelis magellanica & 23 & 3.29 \\
\hline Aratinga sp. & 22 & 3.14 \\
\hline Catamenia analis & 9 & 1.29 \\
\hline Charadrius vociferus & 7 & 1.00 \\
\hline Conirostrum cinereum & 12 & 1.71 \\
\hline Tyrannus melancholicus & 12 & 1.71 \\
\hline Forpus coelestis & 10 & 1.43 \\
\hline Burhinus superciliaris & 7 & 1.00 \\
\hline Columba livia & 7 & 1.00 \\
\hline Aratinga wagleri & 6 & 0.86 \\
\hline Sicalis flaveola & 5 & 0.71 \\
\hline Metriopelia ceciliae & 5 & 0.71 \\
\hline Thaumastura cora & 4 & 0.57 \\
\hline Psilopsiagon aurifrons & 3 & 0.43 \\
\hline Passer domesticus & 3 & 0.43 \\
\hline Rhodopis vesper & 3 & 0.43 \\
\hline Bubulcus ibis & 1 & 0.14 \\
\hline Hirundo rustica & 1 & 0.14 \\
\hline Myrtis fanny & 1 & 0.14 \\
\hline Parabuteo unicinctus & 1 & 0.14 \\
\hline
\end{tabular}


Tabla 3. Distribución de aves en el campus de la UNALM (2003).

\begin{tabular}{|c|c|c|}
\hline Especie & $\begin{array}{c}\mathrm{N}^{\circ} \\
\text { de zonas } \\
\end{array}$ & Frecuencia \\
\hline Zenaida meloda & 42 & 0.93 \\
\hline Columbina cruziana & 41 & 0.91 \\
\hline Dives warszewiczi & 31 & 0.69 \\
\hline Troglodytes aedon & 28 & 0.62 \\
\hline Pyrocephalus rubinus & 24 & 0.53 \\
\hline Crotophaga sulcirostris & 21 & 0.47 \\
\hline Sporophila Velasco & 20 & 0.44 \\
\hline Coereba flaveola & 16 & 0.36 \\
\hline Pygochelidon cyanoleuca & 16 & 0.36 \\
\hline Sturnella bellicosa & 14 & 0.31 \\
\hline Amazilia amazilia & 13 & 0.29 \\
\hline Mimus longicaudatus & 13 & 0.29 \\
\hline Volatinia jacarina & 13 & 0.29 \\
\hline Zenaida auriculata & 13 & 0.29 \\
\hline Zonotrichia capensis & 13 & 0.29 \\
\hline Thraupis episcopus & 12 & 0.27 \\
\hline Aratinga erythrogenys & 11 & 0.24 \\
\hline Coragyps atratus & 10 & 0.22 \\
\hline Forpus coelestis & 9 & 0.20 \\
\hline Camptostoma obsoletum & 7 & 0.16 \\
\hline Charadrius vociferus & 7 & 0.16 \\
\hline Sicalis flaveola & 7 & 0.16 \\
\hline Falco sparverius & 5 & 0.11 \\
\hline Carduelis magellanica & 4 & 0.09 \\
\hline Conirostrum cinereum & 4 & 0.09 \\
\hline Parabuteo unicinctus & 4 & 0.09 \\
\hline Tyrannus melancholicus & 4 & 0.09 \\
\hline Brotogeris versicolurus & 3 & 0.07 \\
\hline Burhinus superciliaris & 3 & 0.07 \\
\hline Circus cinereus & 3 & 0.07 \\
\hline Columba livia & 3 & 0.07 \\
\hline Molothrus bonariensis & 3 & 0.07 \\
\hline Passser domesticus & 3 & 0.07 \\
\hline Psilopsiagon aurifrons & 3 & 0.07 \\
\hline Aratinga wagleri & 2 & 0.04 \\
\hline Athene cunicularia & 2 & 0.04 \\
\hline Glaucidium peruanum & 2 & 0.04 \\
\hline Bubulcus ibis & 1 & 0.02 \\
\hline Icterus graceannae & 1 & 0.02 \\
\hline
\end{tabular}

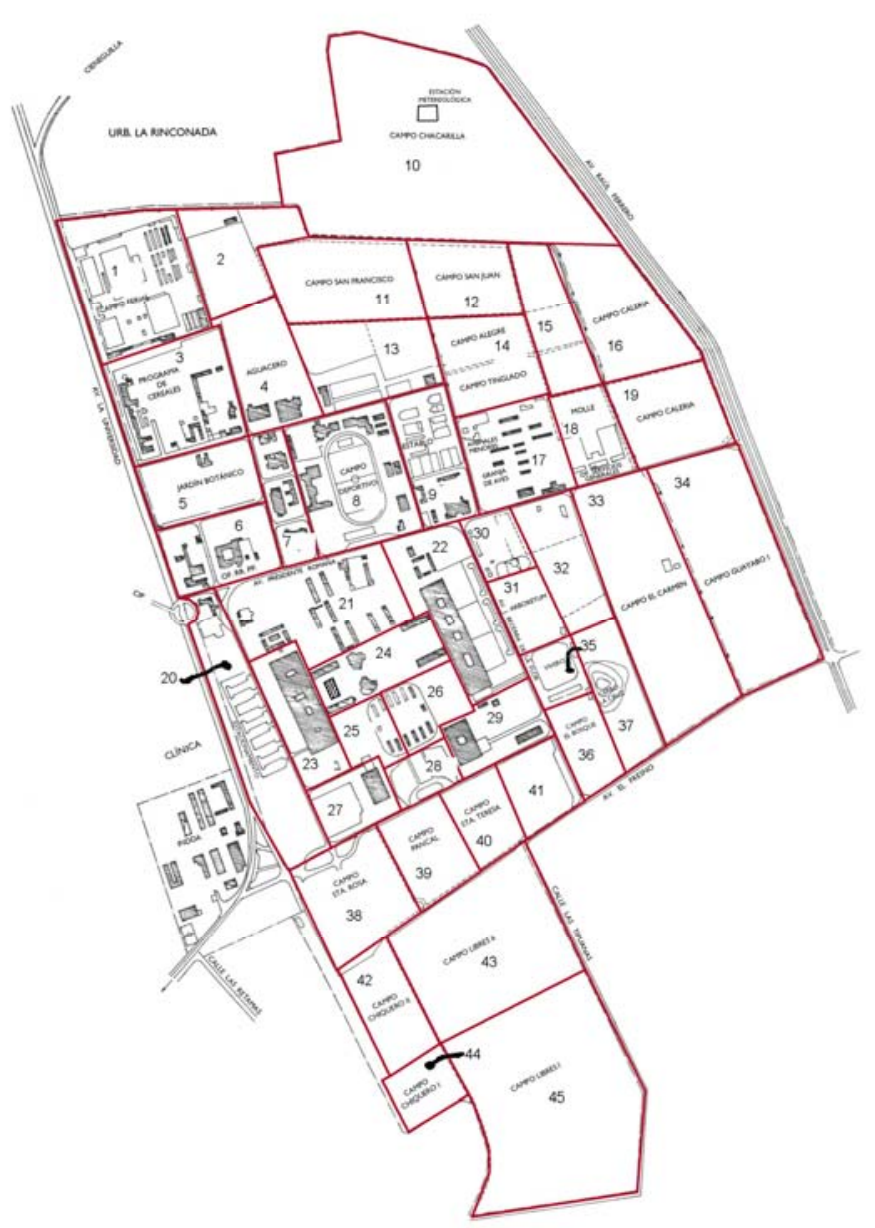

Anexo. Mapa de la Universidad Nacional Agraria La Molina Lima, Perú

Tabla 4. Especies raras vistas en la UNALM en el período 2001-2005.

\begin{tabular}{|c|c|c|c|c|}
\hline & Nombre científico & Nombre común & Familia & Orden \\
\hline 1 & Egretta caerulea & Garza azul & \multirow{3}{*}{\multicolumn{2}{|c|}{\begin{tabular}{ll} 
Ardeidae & Ciconiiformes \\
\cline { 2 - 2 } Pandionidae & Falconiformes \\
Falconidae &
\end{tabular}}} \\
\hline 2 & Pandion haliaetus & Águila pescadora & & \\
\hline 3 & Falco peregrinus & Halcón peregrino & & \\
\hline 4 & Pardirallus sanguinolentus & Gallineta común & \multirow{6}{*}{$\begin{array}{l}\text { Rallidae } \\
\text { Columbidae } \\
\text { Tytonidae } \\
\text { Apodidae } \\
\text { Tyrannidae }\end{array}$} & \multirow{4}{*}{$\begin{array}{l}\text { Gruiformes } \\
\text { Columbiformes } \\
\text { Strigiformes } \\
\text { Apodiformes }\end{array}$} \\
\hline 5 & Columbina minuta & Alfarerita & & \\
\hline 6 & Tyto alba & Lechuza de campanario & & \\
\hline 7 & Chaetura pelagica & Vencejo de chimenea & & \\
\hline 8 & Euscarthmus meloryphus & Mosqueta copetona & & \multirow{6}{*}{ Passeriformes } \\
\hline 9 & Poospiza hispanolensis & Dominiquí común & & \\
\hline 10 & Sporophila luctuosa & Espiguero blanco y negro & \multirow{3}{*}{ Emberizidae } & \\
\hline 11 & Sporophila obscura & Espiguero pardo & & \\
\hline 12 & Sporophila peruviana & Espiguero pico de loro & & \\
\hline 13 & Agelaius icterocephalus & Tordo cabeza amarilla & Icteridae & \\
\hline
\end{tabular}

\footnotetext{
${ }^{1}$ Universidad Nacional Agraria La Molina, Av. La Molina s/n, Lima 12, Perú, f_takano@yahoo.com ${ }^{2}$ Laboratorio de Ecología de Procesos, Departamento de Biología, Facultad de Ciencias, Universidad Nacional Agraria La Molina, Av. La Universidad s/n, Lima 12, Perú, nadia.cci@gmail.com
} 